\title{
Design of Routing Protocols in IoT
}

\author{
Deepa Devassy, J. Immanuel Johnraja
}

\begin{abstract}
IoT is an emerging technology nowadays and it has many applications in our day to day life. Through Internet of Things, millions of devices can be connected to the internet which can communicate each other by exchanging the data. For efficient transmission of packets, proper routing and scheduling alga- rhythms are required. Efficient routing and scheduling strategies ensure proper resource allocation in the network. Most of the IoT system uses the effective packet scheduling and routing concepts to enhance the performance of the network. This paper describes the concepts of various routing and scheduling protocols used in Internet of Things. Working of different protocols and its merits ands merry serial sodas sled in this art icle.
\end{abstract}

\section{Index Terms-Internet of Things, Routing Protocols}

\section{INTRODUCTION}

Millions of devices are getting connected to the world of internet every day, which makes human life so easier. The devices can exchange any kind of information which will be processed and stored in a database. The devices connected in the network is termed as a node which forms the topology of the network. The nodes can be arranged in any structure, so that there should be multiple paths exist among all the nodes. Every node maintains routing information and packets are routed to the destination based on the unique address of the IP header. Routing is significantly important inthe IoT network since the nodes will route the data through the routers and finally it stores in the database or cloud. The selection of best routing algorithm is very much important as far as network throughput is concerned. The concept of IoT is currently related to various technologies like Wireless sensor Network (WSN), Low Power Wireless Personal Area Network (LoWPAN), RFID (Radio Frequency Identification) and Machine to Machine (M2M) Communication[1]. Various types of protocols are being used in all these networksto support the routing of packets. Based on the characteristics of IoT network, the protocols which are working in the wireless network may be taken into the IoT environment. Identify applicable funding agency here. If none, delete this.

Revised Manuscript Received on September 22, 2019.

DeepaDevassy, Research Scholar Karunya Institute of Technology and Sciences Coimbatore, India, dipu.deepa@gmail.com.

J. Immanuel Johnraja, HOD,CSE, Karunya Institute of Technology and Sciences Coimbatore, India, immanueljohnraja@gmail.com.

\section{RELATEDWORKS}

With the current research trend in IoT, every year new proto- cols are getting standardized. There are many protocols defined in every layer of network model which provides different functionality. The network layer protocols [1] are mainly applicable for routing the data in the wired or wire less network. This paper discuss various network layer standards and their application's in different areas so that it can be further expanded to multiple network environments.

\section{LAYERED ARCHITECTURE OF IOT}

IoT architecture[2] can be viewed as a layered architecture and it has five layers. Each layer functionalities are defined as set of protocols. The entire functionality of IoT system is divided into sub functions and each layer performs them. Fig. 1 shows the layered architecture of IoT and each layer is analyzed separately with its features.

\begin{tabular}{|c|}
\hline $\begin{array}{c}\text { Application Layer } \\
\text { (HTTP / CoAP / MQTT) }\end{array}$ \\
\hline $\begin{array}{c}\text { Transport Layer } \\
\text { (TCP / UDP) }\end{array}$ \\
\hline Network Layer \\
(IPv4 / IPv6) \\
\hline Link Layer \\
(Bluetooth /Wi-Fi/ Ethernet/ \\
2G/3G/4G/5G) \\
\hline Physical Layer \\
(Devices / Objects) \\
\hline
\end{tabular}

Fig. 1. Layered Architecture of IoT

\section{A. Physical Layer}

This is the lowest layer in the IoT architecture. This layer mainly deals with the devices and objects connected to the world of IoT.. The devices like sensors, camera and RFID tags fall into this layer. The sensor collects the information such as temperature, humidity etc from the environment and converts this information into signals and passes it on to the devices connected to it. Various types of sensors are available 


\section{Design of Routing Protocols in IoT}

inthemarketforspecificapplications.

\section{B. Link Layer}

Link layer passes the data through the local network connectionlikeWi-Fi,Wi-Maxetc.Protocol sare being defined for different kinds of network which provides different datarate.

\section{Network Layer}

The network layer is responsible for the routing of data packets through the network from source to the destination.IPv4 and IPv6 are the commonly used network layer protocol in IoT application.

\section{Transport Layer}

The transport layer provides end-to end connection between the devices and commonly used transport layer protocols are TCP and UDP.TCP is a connection oriented protocol wherethe connection information is maintained throughout the session. UDP is a connectionless protocol in which the data packets cantakeanyroutetoreachthedestination.

\section{E. Application Layer}

All the user applications run in this layer where different protocols are being used to implement the business model. HTTP is the commonly used application layer protocol and for IoT specific applications other protocols CoAP [2] are also defined.
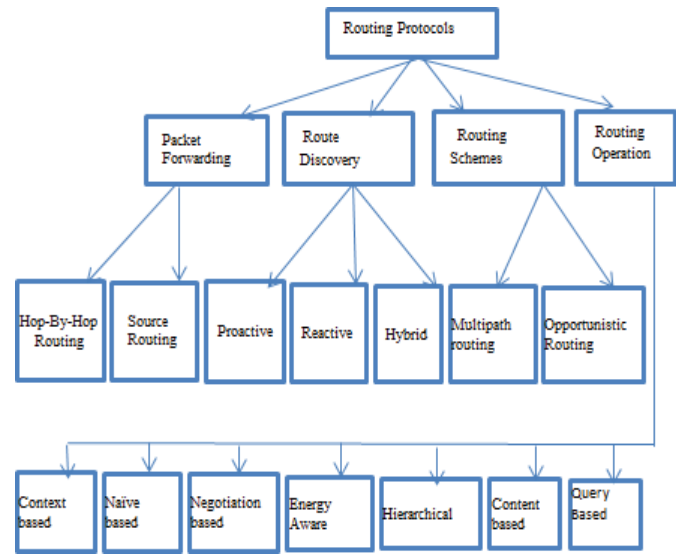

Fig. 2. Classification of Routing Protocols

\section{ROUTING PROTOCOLS IN IOT}

The routing protocols used for wireless network[3] may be applicable to IoT network also.The classification of routing protocols is shown in Fig.2.There exist numerous categories of routing protocols. Each protocol is having its own characteristics which are adaptable in the IoT environment. This section mainly describes the various characteristics of routing protocols applicable to diversenetworks.

\section{A. Packet Forwarding}

Forwarding data packets from source destination cane be done in two different ways, Hop-By-Hop routing[4] and Source routing[5].The details are discussed in the following section.

Hop-By Hop routing: When a packet is travellingthrough the network, it may take different routes with various network devices connected along the path. The devices can be any network connecting devices such as router or gateway .As the packet arrives at each of these device, the device keeps the routing information, accordingly the packet is routed to the destination. The link between two successive network devices is called a hop. Every hop maintains some part of the route it is participating. The routing table at every node contains thenext-hop node and the cost-metric for the destination. Only the destination address is embedded in the data packet.

Source Routing: Insource routing the entire path has been embedded in the packet header itself. Fig.3 depicts how a packet is being routed from a source $\mathrm{S}$ to a destination D.The data packet contains the data and the address of the intermediate routers $\mathrm{A}$ and $\mathrm{B}$ to reach the destination D.It will never follow any other path to reach the destination.

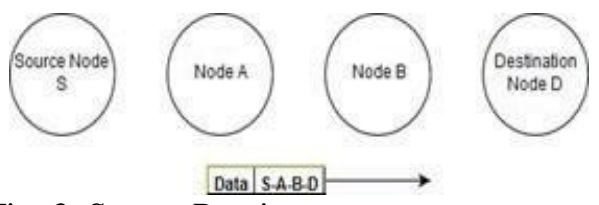

Fig. 3. Source Routing

\section{B. Route Discovery}

The availability of network topological information helps a node to discover its path to reach the destination.Based on the topological information routing protocols can be classified as proactive, reactive and hybrid [6].

Proactive: In proactive protocol, every node is aware about the entire information about the networktopol- ogy before transmitting the data. The routing in- formation is exchanged among all the nodes in the network. This enhances the network performance in terms of latency. However, the exchange of more amount of the routing information, cause more vol- ume of data to be present in the network. Processing of the whole data reduces the lifetime of battery 
attached with the sensor nodes. In this protocol the routing information is periodically exchanged among the other nodes. Therefore ,every node is aware about the network topology and updates routing information in its routing table.

Reactive: Are active protocol exchanges the topology information whenever it is needed. Thus it can save the energy. The path is obtained using a connection establishment process. The nodes are not aware of the network structure, so the routing information is not exchanged among the nodes frequently. Here the main drawback is packets may experience more delay in the network.

Hybrid: It combines the best features of both reac- tive and proactive routing protocols. That is, every node communicates with, neighboring nodes using proactive routing protocols. The communication with far distance node is possible using reactive routing protocol.

\section{Routing Schemes}

The packets can be routed from source to a destination in different ways based on the availability of paths. Based on the path availability from a source to a destination node routing schemes can be classified as follows.

Single-path routing: This is a traditional routing method, which is mostly applicable in wirednet- work. In single path routing[7], the source node construct sais angle tat owe rdsthe sink. The varying link condition may cause routing process inefficient. The examples for single-path routing include DSR, AODV, and DSDV etc.

Multi-path routing: In Multi-path routing[7], multi- ple paths are available between the source tothe destination . So ,the packets can take different routes to reach the destination .This increase the network performance. The main advantages of multi-path routines gore aunt toe raceme oat Dalen connect.

Opportunistic Routing: In this routing, the node clos- est to the the destination is being selected to forward a packet. . This type of routing is mainly appli- cable in wireless sensor networks. Commonly used opportunistic routing protocols[8] include SOAR[9], ExOR[10], ROMER[11]etc.

\section{Routing Operations}

Many of the routing protocols in wireless networks, sensor networks, and Ad Hoc networks can be grad- ually taken into the IoT environment for different applications. According to the goal and the QoS re- quire mentis the routing algorithm can be categorized into different groups. Every day the requirements are changing, and now, it becomes a greater challenge to choose which algorithm to be selected for communication.

Context based routing: The node collects the information from the environment, and uses this data for route selection in IoT. The environmental data suchas temperature, pressure, humid it yet can be taken as measure of finding the energy level of sensor nodes in IoT network. RPL is one of the best examples for context aware routing[12], which uses the concept of different objective functions to learn the arguments from the environment.

Content based routing: In content based routing[10], the data is being transmitted based on the content stored in the database. The database contains the in-formation nsuch as no died, traffic level, MA Caddress and energy level information. The collected data is aggregated and routed based on the content. EECBR [13] and ETERNAL [14] are the routing protocols works on the concept of content basedrouting.

Nave routing: The network uses the concept of flooding[15] in which the source node send request to all the outgoing links and the link propagates this request to its outgoing links until it reaches the destination. Once it reaches the destination, the reply messages is send back to the source node using the reverse path mechanism.

Hierarchical routing: Hierarchical routing[16] is also called as cluster based routing. The nodes forms different clusters or groups in the network. Theclus- ter head is selected based on different criteria. The elected cluster head will forward the data only if the destination belongs to this particular cluster. Energy consumption of the node can be saved using cluster based routing. The protocols such as LEACH[17], FCM[18] are the best examples for energy aware routing protocols.

Query based routing: Query based routing protocols are the one, which falls under energy aware routing protocols[19]. It includes both energy balancing and energy savings. A query is being sent to monitor a specific event happening in the wireless sensor network. To send the query and to get the replay, different strategies are being used.

\section{EXAMPLES OF ROUTINGPROTOCOLS}

In this paper, five different routing protocols have been taken for study and its features have been compared.

Published By:

Blue Eyes Intelligence Engineering \& Sciences Publication

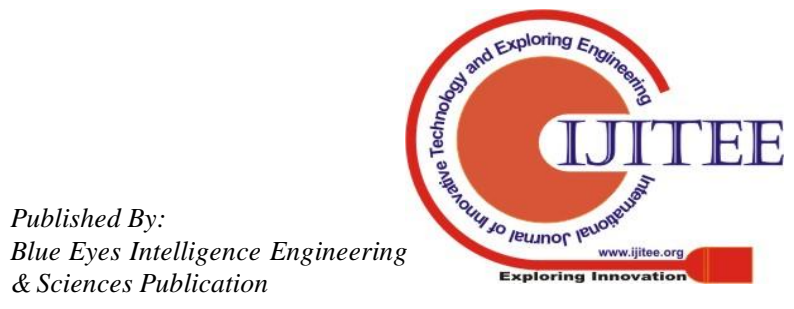




\section{Design of Routing Protocols in IoT}

A. RPL

RPL[20] is the standard routing protocol defined for Low Power and Lossy Networks and nowadays it isbeing used in majority of the IoT applications. RPL uses the concept of Destination Oriented Direct Acyclic Graphs (DODAGs).Every node is ranked with an objective func- tion and it uses different control messages to send the data. When a node receives any DIO message it joins as member in the DAG and forward it to the next node. If a node is already a member of DAG, then it discards the DIO message. DAO control message is used to communicate in the upward direction ie from the node to thegateway.

\section{B. CORPL}

This protocol is an enhancement to the RPL protocol and mainly used in cognitive radio enabled AMI networks. CORPL[21] uses the same concept of DAGwith the con- cept of opportunistic forwarding. Mainly it considers the smart grid application in advanced metering infra structure networks.

\section{CARP}

CARP[22],FBR[23] and EFlood[24] are some of the multi-hop routing protocols used for underwater wireless sensor networks .The hop distance from the sink is being stored by every node in the first step itself. Then a broadcast control packet called PING is transmitted andit is acknowledged with a PONG message by theneig hbors. Then the data is forwarded to the sink through a separate path.

\section{LEACH}

Every node in the network is equipped with a battery which determines the life time of the node in the net work. LEACH[17]is a hierarchical routing protocol, which conservo serener gone debit roper Lyle sterling hen odes. The lifetime of the node can be increased by routing the data through the cluster heads. Many algorithms are proposed to form the clusters and the cluster head can be rotated to impure overhead performance of the net work.

\section{E. ETERNAL}

ETERNAL[14] is a content based routing protocol, in which the data is being routed based on the content than the context. The related data from a network is to be forwarded and thus energy consumption of node can be reduced. ETERNAL algorithm enhances the network life time, and limits the traffic by avoiding the redundant transmission of data. The data coming from various sensors have been collected and aggregated and data is routed based on the content to the destination.

Many IoT routing protocols have been discussed in this paper. These protocols are defined for different kinds of network applications. Table I summarizes the advantage and disadvantages of each of the protocols.

\section{RESULT AND CONCLUSION}

In this survey different routing protocols are analyzed, and it is found that ,for larger networks hierarchical routing protocols are efficient. This protocol reduces the energy consumption of sensor nodes. Hierarchical routing forms, different clusters and only the cluster heads be- come the part of communication. This saves the energy of nodes and increases the life time of network. Flat routing or flooding can be applied for small area networks where the data passes through all the nodes in that network. This leads to more energy consumption at each node and cause more dead no deft cape rain tenet work.

TABLE I COMPARISON OF VARIOUS ROUTING PROTOCOLS

\begin{tabular}{|c|c|c|c|c|}
\hline $\begin{array}{l}\text { Routing } \\
\text { Protocol }\end{array}$ & Application & Methodology & $\begin{array}{l}\text { Context } \\
\text { Aware }\end{array}$ & $\begin{array}{l}\text { Risk } \\
\text { Factor }\end{array}$ \\
\hline RPL & LLN & DODAG & Yes & $\begin{array}{l}\text { Single } \\
\text { point of } \\
\text { failure }\end{array}$ \\
\hline CORPL & $\begin{array}{l}\text { CR } \\
\text { Network }\end{array}$ & $\begin{array}{l}\text { DODAG } \\
\text { with Opportunistic } \\
\text { forwarding }\end{array}$ & Yes & $\begin{array}{l}\text { Each node } \\
\text { maintains } \\
\text { forwarders list }\end{array}$ \\
\hline CARP & $\begin{array}{l}\text { Underwater } \\
\text { WSN }\end{array}$ & $\begin{array}{l}\text { Routing } \\
\text { basedonlink } \\
\text { quality }\end{array}$ & Yes & $\begin{array}{l}\text { Communication } \\
\text { overhead }\end{array}$ \\
\hline LEACH & $\begin{array}{l}\text { WSN } \\
\text { IoT }\end{array}$ & $\begin{array}{l}\text { Hierarchical } \\
\text { Routing }\end{array}$ & Yes & $\begin{array}{l}\text { Cluster head } \\
\text { selection }\end{array}$ \\
\hline ETERNAL & $\begin{array}{l}\text { WSN } \\
\text { IoT }\end{array}$ & $\begin{array}{l}\text { Content } \\
\text { based routing }\end{array}$ & No & $\begin{array}{l}\text { Space } \\
\text { complexity }\end{array}$ \\
\hline
\end{tabular}

\section{REFERENCES}

1) Jorge Granjal, EdmundoMonteiro, and Jorge S Silva "Security for the Internet of Things: A Survey of Existing Protocols and Open Research Issues, "IEEE Communication Surveys and Tutorials,vol.17,pp. 1294 $-1312,2015$. 
2) AlaAl-Fuqaha,MohsenGuizani,MehdiMohammadi,MohammedAledhari ,andMoussaAyyash" Internet of Things: A Survey on Enabling Technologies, Protocols, and Applications,"vol.17,pp. 2347 2376,2015 .

3) https://www.intechopen.com/books/wireless-sensor-networks-technology -and-protocolsmultihop-routing-for-energy-efficiency-

in-wireless-sensor-networks.

4) RonghuiHou,King-Shan Lui, Fred Baker , and Jiandong Li " Hop-by-Hop Routing in Wireless Mesh Networks with Band- width Guarantees, " IEEE Transactions on MobileComputing,vol.11,pp. 264 277, Feb 2012.

5) Giovanni Toso,RiccardoMasiero,PaoloCasari,MaksymKomar,OleksiyKebkal,MicheleZorzi " Revisiting Source Routing for Underwater Networking: The SUN Protocol, "IEEE Access vol.6,pp. $1525-1541,2018$

6) S. Marwaha, Chen KhongTham , D. Srinivasan " Mobile agents based routing protocol for mobile ad hoc networks, " Global Telecommunications Conference, 2002. GLOBECOM '02. IEEE ,March 2003.

7) Pravin R Satav ,Pradip M. Jawandhiya " Review on single- path multi-path routing protocol in manet: A study, " IEEE XPlore,pp.5090-2806 ,June2017.

8) WeiweiChen , Chin-Tau Lea , Shiming He and ZheXuanYuan" Opportunistic Routing and Scheduling for Wireless Networks, " IEEE Transactions on Wireless Communications ,vol.16,pp.320- 331,Jan2017.

9) Eric Rozner,JayeshSeshadri,Yogita Ashok Mehta,andLiliQiu "SOAR: Simple Opportunistic Adaptive Routing Protocol for Wireless Mesh Networks,"' IEEE Transactions on Mobile Com- puting 8(12):1622-1635 December2009.

10) S. Biswas and R. Morris. ExOR: opportunistic multi-hop routing for wireless networks. InProc.of ACM SIGCOMM, Aug. 2005.

11) Y. Yuan,H.Yang,S.H.Wong,S.Lu, and W. Arbaugh "ROMER :Re- silient opportunistic mesh routing for wireless mesh networks. InProc.ofIEEEWiMesh, 2005.

12) Harilton da Silva Arajo ,RaimirHolandaFilho ,Joel J. P. C Rodrigues, Ricardo de A. L. Rabelo, Natanael de C. Sousa ,JosC.C. L. S. Filho and Jos V. V. Sobral , " A Proposal for IoTDynamic Routes Selection Based on Contextual Information,Sensors 2018, 18,353.

13) Chelloug, S.A." Energy-Efficient Content-Based Routing in In- ternet of Things," Journal of Computer andCommunications, 2015 3,9-2.

14) M.DurgaDevi,K.Geetha,K.Saranyadevi" Content Based Routing Using Information Centric Network For IoT, "ScienceDirect Pro- cedia Computer Science, 115 (2017) 707714.

15) SriramSankaran,Ramalingam Sridhar "Modeling and Analysis of Routing in IoT Networks, 'IEEE Conference(CoCoNet'15) Dec. 16-19, 2015, Trivandrum, India.

16) W.T. Tsai, C.V. Ramamoorthy, W.K. Tsai and O. Nishiguchi "An adaptive hierarchical routing protocol, " IEEE Transactions on Computers,vol.38,pp.1059 - 1075,Aug 1989.

17) Asaduzzaman; Hyung Yun Kong "Energy efficient cooperative LEACH protocol for wireless sensor networks, "IEEE Journal of Communications and Networks ,vol. 12,pp. 358 - 365,Aug 2010

18) C Hoang, R Kumar, and SK Panda " Fuzzy c-means clustering protocolfor wireless sensor networks" InIndustrial Electronics (ISIE), 2010 IEEE International Symposium on, pages 34773482. IEEE, 2010.

19) ZhangBing Zhou, Beibei Yao, Riliang Xing, Lei Shu, and ShengrongBu"E-CARP: An Energy Efficient Routing Protocol for UWSNs in the Internet of Underwater Things," IEEE Sensors Journal,vol. 16,pp. 4072 - 4082, June 2016.

20) T. Winter, "RPL: IPv6 Routing Protocol for Low-Power and LossyNetworks," RFC 6550, Accessed on Sep. 2017. [Online].Available:https://rfc-editor.org/rfc/rfc6550.txt

21) Adnan Aijaz,HongiiaSu,andAbdol-Hamid Aghvami " CORPL: ARoutingProtocolforCognitiveRadioEnabledAMINetworks," IEEE Transactions on Smart Grid, vol. 6, pp. 477 - 485,Jan 2015.

22) Stefano Basagni,ChiaraPetrioli,RobertoPetroccia, and Daniele Spaccin "CARP: A Channel-aware routing protocol for under- water acoustic wireless networks," Ad Hoc Networks, vol. 34, pp. 92-104,2015.

23) J.M. Jornet, M. Stojanovic, M. Zorzi, "Focused beam routing protocolfor underwater acoustic networks," in: Proceedings of ACM WUWNet2008, San Francisco, CA, 2008, pp. 7582.

24) D. Shin, D. Hwang, D. Kim, 'DFR: an efficient directional flooding based routing protocol in underwater senso networks,"Wirel.Commun.MobileComput.12(17)(2012)15171527. 